\title{
HOW SEPARABLE IS A SPACE? THAT DEPENDS ON YOUR SET THEORY!
}

\author{
FRANKLIN D. TALL ${ }^{1}$
}

\begin{abstract}
A. Wilansky has raised the question of the productive behaviour of the property of having a countable set, such that each point is a sequential limit point of the set. The set-theoretic consistency and independence of the proposition that this property is preserved by products of $\kappa_{1}$ factors is established.
\end{abstract}

In a recent issue of the American Mathematical Monthly, A. Wilansky [13] points out that, although the product of $2^{\boldsymbol{x}_{0}}$ separable spaces is separable, it need not have a sequentially dense countable subset, even if each factor does. According to Wilansky, a set $D$ is $\kappa$-dense in $X, \kappa$ an infinite cardinal, if there exists a totally ordered set $S$ with $\kappa$ or fewer members, such that each $x \in X$ is the limit of a net in $D$ defined on $S$. He conjectures that the product of fewer than $2^{K}$ spaces, each of which has a $\kappa$-dense subset with $\kappa$ points or less, also has such a subset, but that this does not hold in general for products of $2^{K}$ spaces.

We shall confine ourselves here to the case $\kappa=\boldsymbol{N}_{0}$, for which we can give a complete solution to Wilansky's conjecture. We shall show that the answer depends on what axioms of set theory are assumed in addition to the "usual" ones. By the "usual" ones we mean Zermelo-Fraenkel including the Axiom of Choice, but the same arguments would work for other popular axiomatizations. The case of uncountable $\kappa$ seems much more difficult and less interesting.

Say that a space $X$ is sequentially separable if there is a countable subset $D$ of $X$ such that each point in $X$ is a limit of a sequence from $D$.

Presented to the Society, January 24, 1973; received by the editors February 9, 1973 and, in revised form, October 10, 1973.

AMS (MOS) subject classifications (1970). Primary 54A25, 54B10, 02K05, 54A20; Secondary 04A30, 04A20, 04A10, 02K25, 54D55, 54E 30.

Key words and phrases. Separable, sequential limit, Martin's axiom, topological product, set-theoretic consistency.

1 The preparation of this paper was assisted by grant A-7354 of the National Research Council of Canada. 
It is an easy exercise to verify that sequential separability is equivalent to $\boldsymbol{x}_{0}$-density. We shall establish the following results.

Theorem 1. The product of countably many sequentially separable spaces is sequentially separable.

Theorem 2. It is consistent with the axioms of set theory that $2^{\boldsymbol{N}_{0}}$ be any regular aleph, and that the product of fewer than $2^{\boldsymbol{K}_{0}}$ sequentially sep. arable spaces be sequentially separable.

Theorem 2 is sharp, since a simple counting argument demonstrates that no product of continuum many sequentially separable spaces is sequentially separable if each space has at least two points and its sequential limits are unique. (Singletons and indiscrete spaces respectively prove the necessity of these conditions.) Similarly, again assuming unique sequential limits (as in Hausdorff spaces for example), if $2^{\boldsymbol{N}_{0}}<2^{\kappa}$, no product of $\kappa$ sequentially separable spaces is sequentially separable if each has at least two points. Since $2^{\boldsymbol{N}_{0}}<2^{\boldsymbol{N}_{1}}$ is consistent with the axioms of set theory [6], so is the assumption that no product of uncountably many sequentially separable spaces with unique sequential limits and at least two points is sequentially separable.

To prove Theorems 1 and 2, we use Martin's axiom in its partial order form. For more on Martin's axiom, see [9], [11], [8, Chapter 5], and [12], but we recall the essentials. Let $\mathcal{P}=\langle P, \leq\rangle$ be a partial order. $p, q \in P$ are compatible if there is an $r \in P, r \leq p, r \leq q \cdot \mathcal{P}$ satisfies the countable chain condition if any subset of $P$ with no two members compatible is countable. A subset $T$ of $P$ is dense if for each $p \in P$ there is a $t \leq p, t \in T$. Let $\mathcal{T}$ be a collection of dense subsets of $P . G \subseteq P$ is $\mathcal{T}$-generic if

(1) $p \leq q$ and $p \in G$ imply $q \in G$;

(2) $p, q \in G$ imply there is an $r \in G$ with $r \leq p, r \leq q$;

(3) $G$ meets each $T \in \mathcal{T}$.

Let $\kappa$ be a cardinal. $A_{\kappa}$ is the proposition that for every countable chain condition partial order $\mathcal{P}$ and for each collection $\mathcal{T}$ of fewer than $\kappa$ dense subsets of $P$, there is a $\mathcal{T}$-generic $G \subseteq P . A_{N_{0}}$ is true, for if $\left\{T_{n}\right\}_{n<\omega}$ are dense, pick $p_{0} \in T_{0}, p_{n+1} \in T_{n+1}, p_{n+1} \leq p_{n}$. Then $\{p:$ for some $n$, $\left.p_{n} \leq p\right\}$ is $\left\{T_{n}\right\}_{n<\omega}$-generic. Martin's axiom is the assertion that $\mathbf{A}_{\kappa}$ holds for each $\kappa<2^{\kappa_{0}}$. It is consistent with $2^{\boldsymbol{N}_{0}}$ being any regular uncountable aleph [11].

Theorem 3. $\mathbf{A}_{\kappa}$ implies the product of no more than $\kappa$ sequentially separable spaces is sequentially separable. 
Theorems 1 and 2 follow immediately. The proof of Theorem 3 is a standard forcing argument disguised, as usual, via Martin's axiom - the desired sequence is built up as the union of a generic subset of finite approximations (forcing conditions) to it. It is modeled on the proof in [2, p. 27] that $\mathbf{A}_{\kappa}$ implies that for each collection $\left\{B_{\alpha}\right\}_{a<\kappa}$ of subsets of $\omega$ with any finite intersection of the $B_{\alpha}$ 's infinite, there is an infinite $B \subseteq \omega$, such that for each $\alpha, B-B_{a}$ is finite. In an earlier version of this paper, we claimed that this latter proposition was sufficient to imply that the product of $\leq \kappa$ sequentially separable spaces is sequentially separable. This may well be true, but we are grateful to the referee for finding an as yet unbridged gap in that argument, leading us to substitute this one.

We leave to the reader the task of verifying that if $D_{i}$ is sequentially dense in $X_{i}, 0 \leq i \leq n<\omega$, then $\Pi_{i \leq n} D_{i}$ is sequentially dense in $\Pi_{i \leq n} X_{i}$. We thus shall assume that $\kappa<2^{N_{0}}$ is an infinite cardinal, and that $D_{a}$ is sequentially dense in $X_{a}, \alpha<\kappa$. Put the discrete topology on each $D_{a}$. As the product of no more than $2^{\boldsymbol{K}_{0}}$ separable spaces, $Y=\Pi_{a<\kappa} D_{a}$ is separable [4]. Let $D$ be countable and dense in $Y . D$ is dense in $X=\Pi_{a<\kappa} X_{a}$; in fact we shall show it is sequentially dense, assuming $A_{K}$.

Let $\pi_{a}$ be the projection function from $X$ onto $X_{a}$. Abusing notation, if $f$ is a subset of $\omega \times X$, define

$$
\pi_{a}(f)=\left\{\left\langle n, \pi_{a}(x)\right\rangle:\langle n, x\rangle \in f\right\} .
$$

Let $x \in X, x=\left\langle x_{a}\right\rangle_{a<K^{*}}$. There are sequences $\mathrm{s}_{a}$ from $D_{\alpha}, s_{a} \rightarrow x_{\alpha^{*}}$. Define a partial order $\mathcal{P}=\langle P, \leq\rangle$ as follows.

$P=\{\langle b, H\rangle: b$ is a function from a finite subset of $\omega$ into $D$, $H$ is a finite subset of $\kappa\}$.

$$
\left\langle h^{\prime}, H^{\prime}\right\rangle \leq\langle h, H\rangle \text { if } h^{\prime} \supseteq h, H^{\prime} \supseteq \underline{H} \text {, and for each } a \in H, \pi_{a}\left(h^{\prime}-h\right) \subseteq s_{a} \text {. }
$$

Note that any two members of $P$ with the same first coordinate are compatible. It follows that $\mathcal{P}=\langle P, \leq\rangle$ satisfies the countable chain condition, and that the sets $E_{\alpha}=\{\langle h, H\rangle: \alpha \in H\}$ are dense in $P$.

Let $F_{n}=\{\langle h, H\rangle: n \in$ domain $h\}$. We shall prove that each $F_{n}$ is dense. Let $\langle h, H\rangle \in P$ be given. $U_{k}=\left(\Pi\left\{D_{a}: \alpha<\kappa, \alpha \notin H\right\}\right) \times\left(\Pi\left\{\left\{s_{a}(k)\right\}: a \in H\right\}\right)$ is open in $Y$, each $k<\omega$. Suppose $n \notin$ domain $h$. Pick $d \in D \cap U_{n}$. Let $h^{\prime}=h \cup\{\langle n, d\rangle\}$. Then $\langle h, H\rangle \geq\left\langle h^{\prime}, H\right\rangle \in F_{n^{\prime}}$.

We now are in a position to construct the desired sequence out of $D$ converging to $x \in X$. Let $G$ be $\left(\left\{E_{a}\right\}_{a<K} \cup\left\{F_{n}\right\}_{n<\omega}\right)$-generic. Let $g=$ $\bigcup\{h$ : for some $H,\langle h, H\rangle \in G\}$. By compatibility, $g$ is a function. Its do- 
main is all of $\omega$, since $G$ meets each $F_{n}$. Thus $g$ is a sequence from $D$. We will show its $\alpha$-projections converge to $x_{a}$ so that it converges to $x$. A standard argument yields that for each $\alpha, \pi_{\alpha}(g)-s_{\alpha}$ is finite. Namely take $\left\langle h_{0}, H_{0}\right\rangle \in G$ such that $a \in H_{0}$. Then $\pi_{\alpha}(g)-s_{a} \subseteq \pi_{\alpha}\left(h_{0}\right)$, and for given any $\left\langle h_{1}, H_{1}\right\rangle \in G$ there is an $\left\langle h_{2}, H_{2}\right\rangle \in G$ with $\left\langle h_{2}, H_{2}\right\rangle \leq$ both $\left\langle h_{0}, H_{0}\right\rangle$ and $\left\langle h_{1}, H_{1}\right\rangle$. Then $\pi_{a}\left(h_{2}-h_{0}\right) \subseteq s_{a}$, so $\pi_{a}\left(h_{1}-h_{0}\right) \subseteq s_{a}$. Since $\left\langle h_{1}, H_{1}\right\rangle$ was arbitrary, $\pi_{a}\left(g-h_{0}\right) \subseteq s_{\alpha}$, so $\pi_{\alpha}(g)-s_{\alpha} \subseteq \pi_{\alpha}\left(h_{0}\right)$. Thus $\left\{n: \pi_{\alpha}(g)(n) \neq s_{\alpha}(n)\right\}$ is finite. Since $s_{\alpha} \rightarrow x_{a}$, so does $\pi_{\alpha}(g)$.

The special case of whether the product of $\kappa_{1}$ copies of the two point discrete space is sequentially separable is particularly interesting. We have now shown this question to be independent of the axioms of set theory. It is also equivalent to a well-known problem in topology.

Theorem 4. $2^{\omega_{1}}$ is sequentially separable if and only if there is a separable normal nonmetrizable Moore space.

The proof is obtained by putting together a number of known results.

Rothberger [10, Theorem 6] proves the equivalence of $2^{\omega_{1}}$ being sequentially separable with the existence of an uncountable set $X$ of reals such that in the subspace topology, every subset of $X$ is an $F_{\sigma}$. (In Rothberger's terminology, the former proposition is stated as "there is a denumerable base for the dyadic functions on any set of power $\kappa_{1}$ ".). The latter proposition is equivalent to the existence of a separable normal nonmetrizable Moore space by Bing [1, Example E] plus Heath [7, Theorem 1]. Bukovsky [3] exhibits a model of set theory in which $2^{N_{0}}=2^{\boldsymbol{N}_{1}}$, but in which every separable uncountable metric space has a non-Borel subset. Thus we obtain

Corollary 5. It is consistent with the axioms of set theory that $2^{\boldsymbol{N}_{0}}>$ $\boldsymbol{\kappa}_{1}$, but $2^{\omega_{1}}$ is not sequentially separable.

Foland and Kirk [5] have also worked on Wilansky's conjecture independently of the author and roughly simultaneously. Their results for uncountable cardinals can also be gotten by a straightforward extension of our methods. We need the generalized continuum hypothesis, as they do, to achieve positive results for uncountable $\kappa$, but they use it for the countable case where it is not necessary.

\section{REFERENCES}

1. R. H. Bing, Metrization of topological spaces, Canad. J. Math. 3 (1951), 175-186. MR 13, 264.

2. D. D. Booth, Countably indexed ultrafilters, Thesis, University of Wisconsin, Madison, Wis., 1969. 
3. L. Bukovsky, Borel subsets of metric separable spaces, General Topology and its Relations to Modern Analysis and Algebra II, Proc. Second Prague Topological Sympos., 1966, Academia, Prague; Academic Press, New York, 1967, pp. 83-86. MR 38 \#51.

4. W. W. Comfort, A short proof of Marczewski's separability theorem, Amer. Math. Monthly 76 (1969), 1041-1042. MR 40 \#1993.

5. N. E. Foland and R. B. Kirk, Products of spaces with m-dense subsets (preprint).

6. K. Gödel, Consistency-proof for the generalized continuum hypothesis, Proc. Nat. Acad. Sci. U.S.A. 25 (1939), 220-224.

7. R. W. Heath, Screenability, pointwise paracompactness and metrization of Moore spaces, Canad. J. Math. 16 (1964), 763-770. MR 29 \# 4033.

8. I. Juhász, Cardinal functions in topology, Mathematical Centre, Amsterdam, 1971.

9. D. Martin and R. M. Solovay, Internal Cohen extensions, Ann. Math. Logic 2 (1970), 143-178. MR 42 \#5787.

10. F. Rothberger, On some problems of Hausdorff and of Sierpiniski, Fund. Math. 35 (1948), 29-46. MR 10, 689.

11. R. M. Solovay and S. Tennenbaum, Iterated Cohen extensions and Souslin's problem, Ann. of Math. (2) 94 (1971), 201-245. MR 45 \#3212.

12. F. D. Tall, An alternative to the continuum hypothesis and its uses in general topology (preprint).

13. A. Wilansky, How separable is a space? Amer. Math. Monthly 79 (1972), 764-765.

DEPARTMENT OF MATHEMATICS, UNIVERSITY OF TORONTO, TORONTO, ONTARIO MSS 1A1, CANADA 